\title{
Energy efficient adaptive cellular network configuration with QoS guarantee
}

\author{
Pierpaolo Piunti*, Cicek Cavdar ${ }^{\dagger}$, Simone Morosi* $^{*}$ Kaleab Ejigayehu Teka ${ }^{\dagger}$, Enrico Del Re ${ }^{*}$ and Jens Zander $^{\dagger}$ \\ *Department of Information Engineering, University of Florence, Florence, Italy \\ Email: pierpaolo.piunti@unifi.it, simone.morosi@unifi.it, enrico.delre@unifi.it \\ ${ }^{\dagger}$ Communication Systems Department, KTH Royal Univesity of Technology, Kista, Sweden \\ Email: cavdar@kth.se, kaleab.teka@masterschool.eitictlabs.eu, jenz@kth.se
}

\begin{abstract}
Cellular network energy optimization is driven by different factors, namely transmission power and activity of the base stations. Quality of Service $(\mathrm{QoS})$ of users is determined by received power, interference and bandwidth allocation and cannot be neglected. In existing studies, such drivers are treated separately, i.e., bandwidth allocation is fixed while power consumption is a variable to be optimized. This paper proposes a novel optimization framework which is aimed at minimizing the power consumption in cellular networks while affording a minimum bit rate for each mobile terminal by jointly considering energy consumption and QoS drivers. Mixed Integer Quadratic Programming (MIQP) based optimization framework solves the problems of the determination of the user association, the bandwidth allocation, the identification of the active base stations and their transmission power, guaranteeing also a requested service rate for each user. The proposed solution is shown to allow power savings of up to $60 \%$, very close to the optimum lower bound, when the traffic is below the $35 \%$ of the maximum load.
\end{abstract}

\section{INTRODUCTION}

In the internet era, mobile traffic is becoming one of the dominating contributors of the increasing data rates. Internet traffic is envisaged to grow approximately by a factor of 1.7 each year [1] while the mobile operator average revenue per user (ARPU) tends to decline due to the market saturation and the high infrastructure costs. In order to cope with this revenue gap, mobile operators are looking for methods to reduce their cost with no loss in the quality of service (QoS). However, capacity growth comes together with an increase in power consumption. From sustainability perspective, there are currently more than 4 million base stations in the world consuming $60 \mathrm{TWh}$ energy per year corresponding to $80 \%$ of the energy that is spent for mobile telecommunications [2]. Mobile networks are dimensioned according to the peak data rates in order to satisfy the user requirements at busy traffic hours; however, the infrastructure capacity is underutilized most of the time, calling for adaptive base station activation and deactivation mechanisms according to user and traffic distribution to reduce power consumption: as a matter of fact, capacity must be available when and where the user demands for it, but base stations do not have to be always on.

A large number of studies have been recently carried out focusing on traffic adaptive activation of radio resources to save energy in cellular networks. Since mobile networks are dimensioned according to peak traffic hours, a set of BSs can be put in sleep mode when the traffic load is light, e.g., during the night hours. Using this solution, the BS activity could be adapted to the traffic demand avoiding the energy wasted due to dimensioning for peak demand [3]. BS sleep is a critical issue because when a deactivation decision is taken, some coverage holes could arise in the area served by that BS. Therefore a layer of BSs is assumed to serve as coverage layer and load adaptive cell sleep strategies are not considered for those BSs. For traffic fluctuations happening between day and night, i.e. according to a coarse time granularity, macro sleep or deeper sleep can be enabled under this assumption. However, for medium to high traffic hours deep sleep is not possible. Therefore, in the time domain finer granularity of sleep mode has been introduced. Such a strategy, identified as cell discontinuous transmission (DTX) [4], is enabled by the particular structure of the LTE frame and allows the transceiver deactivation (sleep mode) during the idle time slots. Thus, signaling symbols can be transmitted and cell coverage is not affected. Cell DTX feature does not require specific decisions to be taken regarding when to sleep and traffic offloading [4]. However, the saving that can be achieved is limited compared to deep long term sleep. For the long term sleep, in order to maximize the energy savings, the association between users and BSs can be considered as an optimization variable together with bandwidth allocated to each user [5]. As a matter of fact, energy can be saved by increasing the bandwidth per user and, consequently, reducing the BS transmission power if the target datarate per user is fixed [6]. Such a solution is known as bandwidth expansion mode (BEM) and can be applied when resource utilization is light, i.e. in low load conditions. Another radio resource management (RRM) solution for energy saving is power control, which aims at minimizing the BS transmission power with a given QoS target for the served users. Note that the benefit of power control is not only in the energy reduction but also in neighbour cell interference management. In particular, in [7] authors propose an optimization framework to maximize the quantity of transmitted bits per energy unit. Such a solution looks at the energy efficiency maximization, but does not care about a QoS target for each user. Moreover, in [8], transmission power for an OFDM communication is minimized without considering any constraint on power model.

By combining some of these approaches, different opti- 
mization solutions have been proposed. A pricing algorithm is evaluated in [9] to solve user association problem and minimize area power consumption, while considering interference: therein, mobile terminal rates are fixed and bandwidth is equally allocated to users regardless of their received signal strength. In [10] power supply per LTE frame is minimized by assigning an opportune transmission power and rate for each link, without considering the mapping problem and a minimum rate value to ensure QoS. Quality of service constraints are considered in [11], [12]. In [11] BS transmission power and user rate are optimized in order to maximize the energy efficiency in terms of "bit per Joule". The formulation considers a single cell scenario and the effect of neighbouring interference and users mapping are not considered. In [12] a two step algorithm aiming at minimizing energy consumption by opportunely assigning subcarriers and power to users is proposed. Such a solution considers a very simple bandwidth division among served users and does not take advantage of BEM in order to reduce the unfairness in the perceived datarate.

In this study, a more realistic strategy is investigated: a given service rate is guaranteed to mobile terminals; if sufficient bandwidth resources are available, mobile users can obtain higher rates than the target value since their received power must be greater than the terminal sensitivity threshold. Moreover, the bandwidth blocks are assigned not uniformly, but according to the spectral efficiency of the overall user associations, saving more resources for the users experiencing lower signal quality and including the benefits provided by BEM. Since spectral efficiency depends on the interference as well as on the user associations, the solution of the optimization problem is not trivial. Hence, an optimization framework is introduced which is based on Mixed Integer Quadratic Programming (MIQP): within this framework the user associations are solved together with the decision variables for the base stations' activity. Moreover, also the user bandwidth and rate assignments as well as transmit power of each active base station are determined. This approach utilizes a snapshot model where the problem is solved for a given time while all users are transmitting together. Moreover, the cell switch off is possible only if another layer guarantees the coverage of the area. This work focuses on the capacity layer, assuming the existence of a coverage layer. This assumption is justified by the $5 \mathrm{G}$ vision that separates control and data layer in cellular networks for green network operation [13]. In order to evaluate the framework performance, we have used two benchmarking solutions: as an upper bound, a conventional power control algorithm is used whereas, as a lower bound, MIQP model is solved to find minimum power consumption, guaranteeing the requested user rates only if interference between neighbour cells could be neglected.

The paper is organized as follows. Sec. II describes the system model and the scenario. In Sec. III the optimization problem is formulated. Sec. IV describes the evaluated energy saving strategies. Sec. V discusses the simulation results and, finally, Sec. VI concludes the work.

\section{THE SYSTEM MODEL}

A typical LTE system deployment is considered with a given bandwidth and a set of resource blocks. This study focuses on the capacity layer, assuming that the coverage condition is guaranteed by another layer of the cellular deployment. In the considered deployment, a set of omnidirectional base stations (BSs) provides radio access to a certain number of user equipments (UEs). Each base station must allocate the available bandwidth resources among the associated transmitting users by assigning the proper amount of physical resource blocks (PRBs) and guaranteeing their satisfaction in terms of bitrate. UEs request a constant bitrate and will be served at the same time.

Let $\mathcal{B}=\left\{B S_{1}, \ldots, B S_{N}\right\}$ and $\mathcal{U}=\left\{U E_{1}, \ldots, U E_{M}\right\}$ be the set of $N$ deployed base stations and the set of $M$ users which have to be served respectively. The binary variable $x$ models the association between BSs and UEs, such that:

$$
x_{i j}=\left\{\begin{array}{ll}
1 & \text { if UE } j \text { is served by BS } i \\
0 & \text { otherwise }
\end{array} \quad i \in \mathcal{B}, j \in \mathcal{U}\right.
$$

Assuming $\pi_{i j}$ is the power assigned for transmission between BS $i$ and UE $j$, and $w_{i j}$ is the bandwidth assigned by BS $i$ to UE $j$, the datarate achieved by UE $j$ is:

$$
\rho_{j}=\sum_{i \in \mathcal{B}} x_{i j} w_{i j} \log _{2}\left(1+\gamma_{i j}\right)
$$

where $\gamma_{i j}$ is the SINR between BS $i$ and UE $j$. Transmission power of each BS $i$ is calculated as $P_{i}=\sum_{j \in \mathcal{U}} \pi_{i j} x_{i j}$. Therefore, the SINR $\gamma_{i j}$ is

$$
\gamma_{i j}=\frac{\pi_{i j} \sigma_{i j} x_{i j}}{\frac{w_{i j}}{W}\left(\sum_{k=1}^{N} P_{k} \sigma_{k j} \zeta_{k}\left(1-x_{k j}\right)+W N_{0}\right)}
$$

where $\sigma_{i j}$ is the channel gain between BS $i$ and UE $j, W$ is the total available bandwidth at a BS and $N_{0}$ is the noise spectral density. Note that in (3) the received interference is weighted by the effective fraction of bandwidth assigned to the UE. Such a choice is justified by the need to consider the effect of allocating different portions of bandwidth to each UE. The activity status of each BS is modelled by the binary variable $\zeta$, such that:

$$
\zeta_{i}=\left\{\begin{array}{ll}
1 & \text { if BS } i \text { is active } \\
0 & \text { if BS } i \text { is in SLEEP mode }
\end{array} \quad i \in \mathcal{B}\right.
$$

\section{PROBLEM FORMULATION}

Given the system defined in Sec. II, the problem is to minimize the global power consumption. Therefore, the op- 


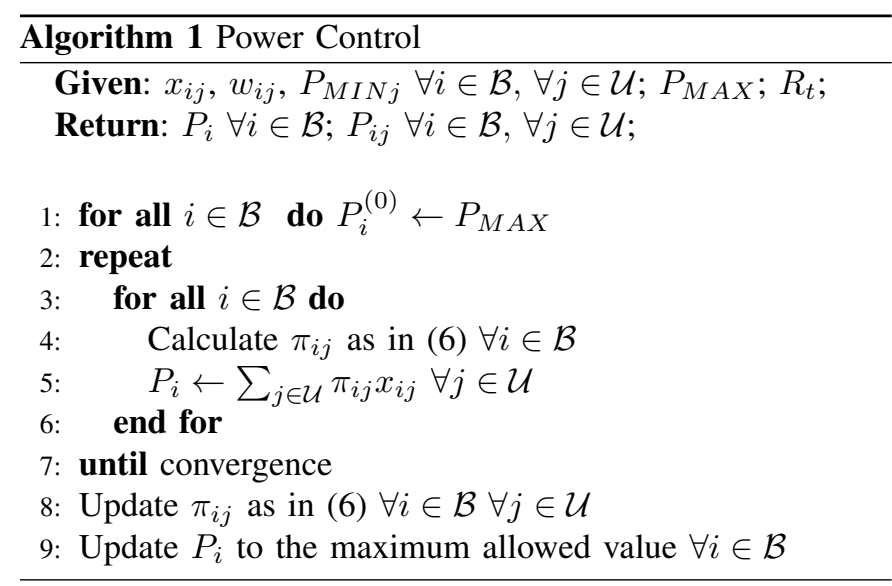

timization problem is formulated in (5a)-(5h).

$$
\begin{aligned}
& \underset{\pi, x, \zeta}{\arg \min } \sum_{i=1}^{N}\left[\left(a \sum_{j=1}^{M} \pi_{i j} x_{i j}+P_{0}\right) \zeta_{i}+\left(1-\zeta_{i}\right) P_{\text {sleep }}\right] \\
& \text { s.t. } \quad \sum_{j=1}^{M} x_{i j} \leq N_{P R B} \quad \forall i \in \mathcal{B} \\
& \sum_{i=1}^{N} \sum_{j=1}^{M} x_{i j}=M \\
& \sum_{i=1}^{N} x_{i j}=1 \quad \forall j \in \mathcal{U} \\
& c_{i j} \leq \frac{\pi_{i j} \cdot \sigma_{i j}}{P_{M I N j}} \quad \forall i \in \mathcal{B} \quad \forall j \in \mathcal{U} \\
& c_{i j}-x_{i j} \geq 0 \quad \forall i \in \mathcal{B} \quad \forall j \in \mathcal{U} \\
& \zeta_{i} \leq x_{i j} \quad \forall j \in \mathcal{U} \quad \forall i \in \mathcal{B} \\
& \sum_{j=1}^{M} \pi_{i j} \leq P_{M A X} \quad \forall i \in \mathcal{B}
\end{aligned}
$$

The objective function is the global power consumption, which is calculated as in [14]; parameters $a, P_{0}$ and $P_{\text {sleep }}$ are the slope of the dynamic consumption, the fixed consumption and the sleep mode consumption respectively. Constraint (5b) is for the BS capacity limitation, since a BS cannot assign more than available bandwidth elements $N_{P R B}$. Constraints (5c) and (5d) ensure that each UE must be covered by at least one BS and can be connected to only one BS at a time. Constraint (5e) is the key for assuring the QoS. The binary variable $c_{i j}$ equals to 0 if $\pi_{i j} \sigma_{i j} \leq P_{M I N j}$. For a given $\mathrm{UE}, c_{i j}$ will define the set of potential BSs that can provide the minimum received power, $P_{M I N j}$. Then, by the help of constraint (5f), only one of the BSs in this set is selected. The activity status of a base station is linked to the user associations by constraint $(5 \mathrm{~g})$. Finally, constraint $(5 \mathrm{~h})$ set the limit on BS transmission power.
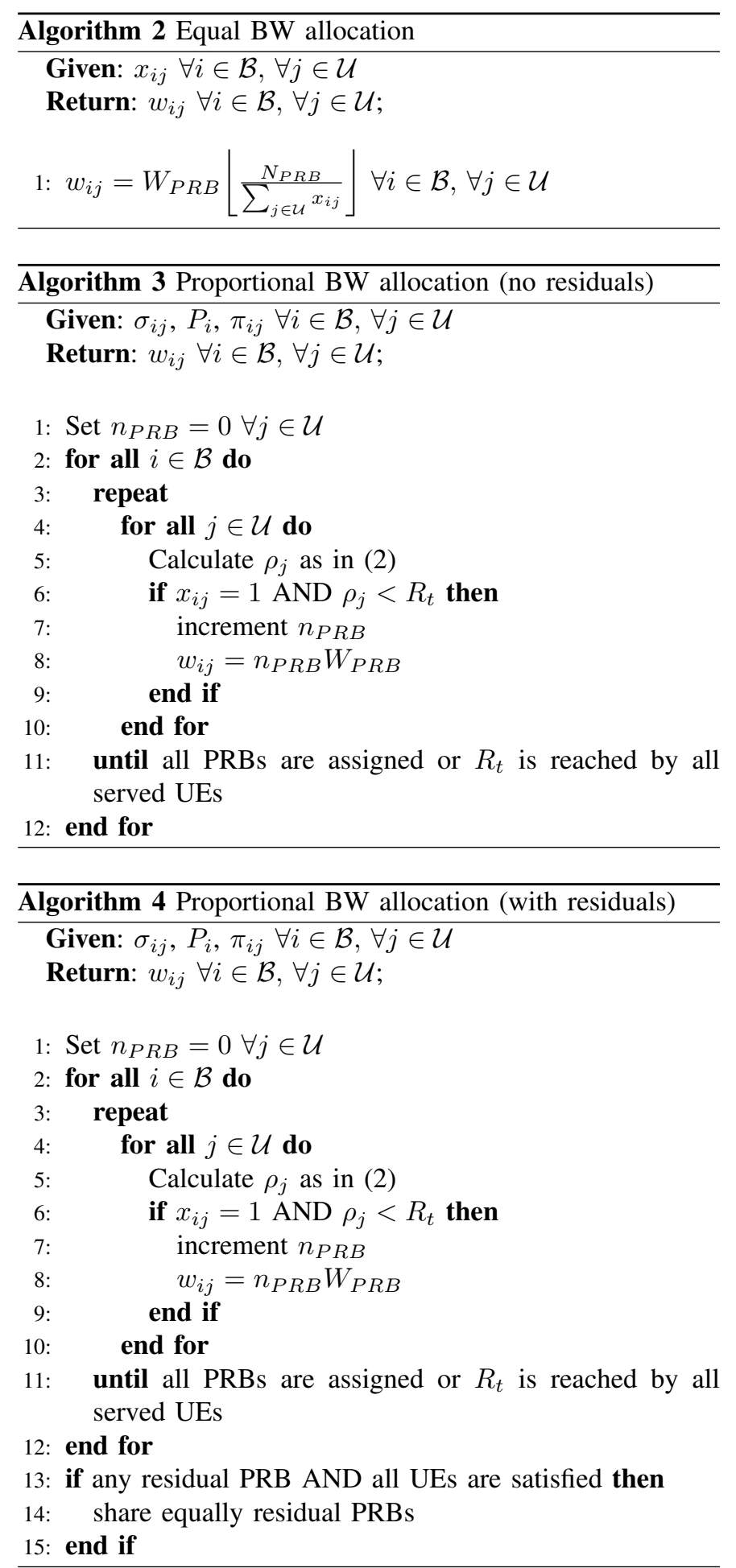

\section{NETWORK ADAPTATION SOLUTIONS}

\section{A. Power control}

As described in Sec. I, power control is a well known solution to decrease the global energy consumption by acting on the reduction of intercell interference. In this work a modified version of the power control algorithm presented in [15] is considered in order to extend its solution to a multichannel 

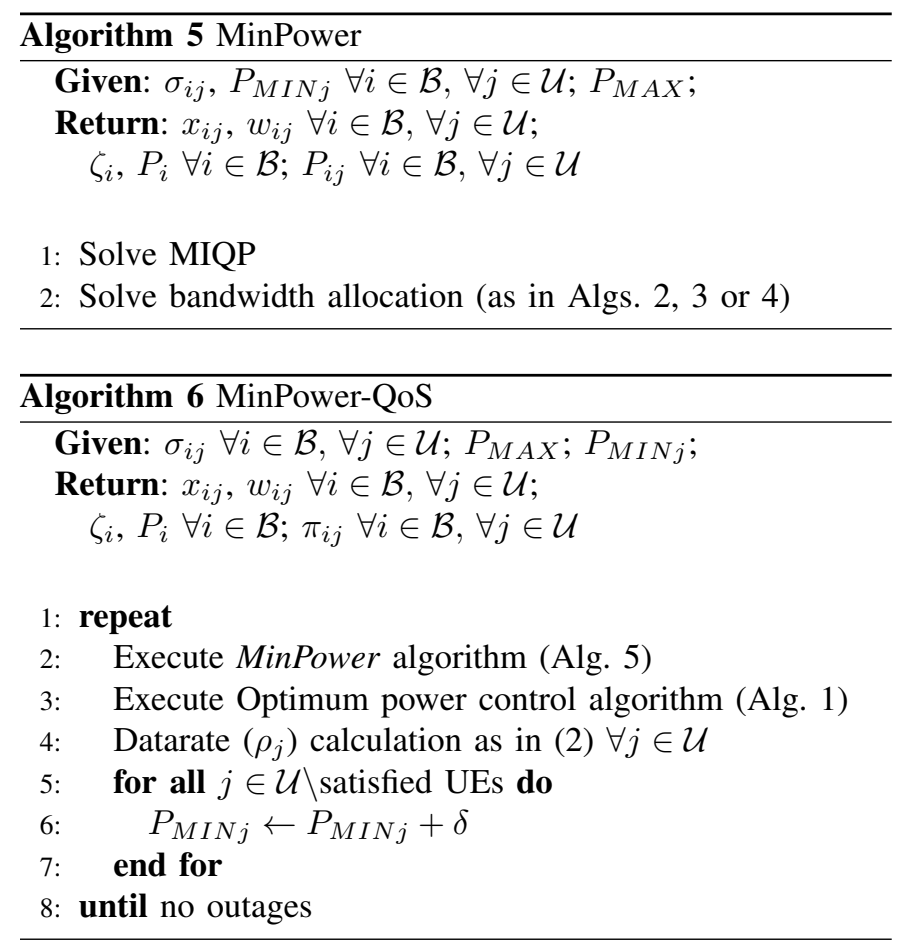

TABLE I

SIMULATION PARAMETERS

\begin{tabular}{lc}
\hline Parameter & Value \\
\hline Deployment & $19 \mathrm{BS}, \mathrm{ISD}=500 \mathrm{~m}, \operatorname{hexagonal}$ grid, wrap-around \\
Path loss & $L=15.3+37.6 \log (d)$ (3GPP Typical Urban) \\
Shadow fading & std dev $8 \mathrm{~dB}$ \\
Indoor loss & $20 \mathrm{~dB}$ \\
Bandwidth & $5 \mathrm{MHz}(25 \mathrm{PRBs})$ \\
Carrier frequency & $2 \mathrm{GHz}$ \\
Max BS $P_{T X} X$ & $20 \mathrm{~W}$ \\
UE sensitivity & $-90 \mathrm{dBm}$ \\
Noise PSD & $-174 \mathrm{dBm} / \mathrm{Hz}$ \\
Target user datarate & $512 \mathrm{Kbps}$ \\
Power consumption & $a=4.7 P_{0}=130 \mathrm{~W} P_{\text {sleep }}=13 \mathrm{~W}$ \\
\hline \hline
\end{tabular}

scenario with minimum and maximum power constraints ${ }^{1}$. As shown in Alg. 1, this power control algorithm takes as input a UE-BS association and a bandwidth assignment for each UE and it iteratively obtains the optimum BS transmission power which is able to guarantee the target datarate for each UE. The core of the optimum power control algorithm is to calculate at each iteration $n$ the power transmitted by a BS to a certain $\mathrm{UE}$ as

$$
\pi_{i j}^{(n)}=\frac{w_{i j} 2^{\frac{R_{t}}{w_{i j}}}}{W \sigma_{i j}}\left(\sum_{k \in \mathcal{B}} P_{k}^{(n-1)}\left(1-x_{k j}\right) \sigma_{k j}+W N_{0}\right)
$$

where $R_{t}$ is the target datarate, i.e. the QoS constraint for each UE. The initial condition is such that $\sum_{j} \pi_{i j}^{(0)}=P_{M A X}$ for all $i \in \mathcal{B}$. Note that the power assigned to a BS, i.e. $P_{i}$, cannot be greater than the maximum allowed power $P_{M A X}$; in that case the power $P_{M A X}$ is divided equally among each

\footnotetext{
${ }^{1}$ For the proof of convergence in iterative power control, please see [15], pp. 163-171.
}

UE to indicate the UEs under outage. Moreover, the received power for each UE $j$ cannot be smaller than the sensitivity $P_{M I N j}$; in that case the power which is transmitted by a BS to a certain UE is adjusted by $\pi_{i j}=\max \left(\frac{P_{M I N j}}{\sigma_{i j}} ; \pi_{i j}\right)$.

\section{B. Bandwidth adaptation}

Bandwidth assignment to each served UE is a task that can be solved in different ways by radio network operators (RNOs) depending on their policies. The simplest way to do it is to equally split the available bandwidth among all connected UEs, as in Alg. 2: in this strategy the UE performance depends only on the signal quality for a given PRB bandwidth $\left(W_{P R B}\right)$ and a given amount of PRBs at the BS $\left(N_{P R B}\right)$. Moreover, a RNO could give more priority to the UE experiencing the best channel conditions in order to maximize the throughput. The distribution of bandwidth resources becomes important especially when the resources are limited. When an energy saving strategy is applied and the network capacity is reduced by putting a set of BSs in sleep mode, each served UE should be guaranteed a level of QoS.

Bandwidth assignment strategy has an impact also on the energy saving potentials. Different policies can result in different number of BSs to be put in sleep mode when bandwidth is shared among a set of UEs. When the amount of PRBs is equally shared among UEs, the rate provided to the UEs experiencing better channel conditions will be higher than the others. Alg. 3 and Alg 4 present a possible solution to assign bandwidth in order to achieve fairness among UEs in terms of QoS, i.e., user data rates. In particular, Alg. 3 assigns just the needed amount of bandwidth allowing each UE to reach the target QoS. On the other hand, Alg. 4 equally splits the residual PRBs in order to increase the UE performance. Note that both algorithms converge to the same behaviour for high number of connected UEs or weak channel quality.

In this paper the focus is not on bandwidth assignment strategies but on the evaluation of the impact of the bandwidth management on the power savings.

\section{Network energy optimization guaranteeing QoS}

In order to introduce a higher power saving in cellular networks, a new optimization framework is proposed. The problem is iteratively solved for three variables: association between BS and UE, bandwidth assignment and power allocation. In particular, an opportune BS-UE association allows saving power by increasing the number of BSs that are not serving traffic and that can be deactivated or put into sleep mode. By joint bandwidth and power allocation a further gain is obtained by reducing transmission power and decreasing the intercell interference. BSs deactivation and power reduction are allowed only if no outage is introduced, i.e. the target QoS is satisfied for each served UE.

The optimization framework is composed of (i) MIQP solver to optimize the UE to BS mapping and the active BSs set, (ii) bandwidth allocation scheme and (iii) power control algorithm which is used to control the feasibility of the UE to BS mapping found in (i) and to identify the outages. The 
MIQP optimization refers to the problem formulated in (5a)(5h). The output of this step is the mapping $x_{i j}$ between BSs and UEs, the power transmitted by each BS to each connected UE $\pi_{i j}$ and the set of active BS $\zeta_{i}$. Then in the second step the bandwidth is allocated to each UE by the respective serving BSs. The bandwidth allocation is performed following Alg. 2, Alg. 3 or Alg. 4.

The MIQP model is solved by IBM ILOG CPLEX ${ }^{\circledR}$ solver. Since the model cannot manage directly the QoS for each UE because of its non-linearity, two approaches are proposed in order to avoid outages. These approaches are (i) Power consumption minimization assuming an interference controlled scenario (MinPower); (ii) Iterative power consumption minimization to guarantee QoS (MinPower-QoS). The MinPower scenario assumes a good planning or a perfect intercell interference cancellation (ICIC) solution, but it could be also the reference condition for rural areas. In that case the rate of each user is only dependent on the signal to noise ratio (SNR), so the only interesting variable is the power received by the serving BS. If interference cannot be neglected, MinPower, as shown in Alg. 5 cannot guarantee the required quality of service and some outages could arise. For that reason, this algorithm represents an optimum lower bound for the network optimization in terms of global power consumption. In order to avoid the datarate outages MinPower-QoS is introduced. MinPower-QoS is presented in Alg. 6 and combines the optimum power control and the MinPower approaches in an iterative framework. In particular MinPower is executed in order to obtain the optimum set of active BSs, the optimum mapping and the bandwidth assignment and minimize the power consumption, while the feasibility of this solution is controlled by the Power Control as shown in Alg. 6. If some datarate outages occur, the received power of the users which do not satisfy the target QoS is iteratively increased by a $\delta$ value in order to select a better mapping and active BSs set.

\section{Simulation Results}

In order to evaluate the power consumption savings due to the base station sleep mode introduction and the transmission power adaptation, the proposed solution, namely the MinPower- $Q o S$ is compared to upper bound and lower bound solutions. As an upper bound, Closest BS mapping follows the Alg. 1 based on power control with closest BS mapping and equal bandwidth assignment to each UE. BSs which are not serving any UE are put into sleep mode. As a lower bound, the optimum solution MinPower is used. Moreover, referring to MinPower-QoS, different bandwidth allocation strategies have been considered in order to show the bandwidth effect on the power optimization. Therefore MinPower- $Q o S$ eq $b w$ follows the bandwidth allocation solution described in Alg. 2, i.e. bandwidth is equally divided among UEs; MinPowerQoS no res follows Alg. 3, i.e. UEs experiencing the worst channel conditions are assigned more bandwidth blocks. After achieving the target rate requirements for each UE, the residual PRBs are not assigned; MinPower-QoS res follows Alg. 4, i.e. residual PRBs are equally split among UEs allowing higher data rates than what is required. Simulation parameters are reported in Table I [16]. For the sake of simplicity omnidirectional antennas have been considered instead sectored sites. The considered sleep mode power value is in line with evaluations done in [17]. In each iteration of MinPower-QoS, if a UE cannot achieve the datarate target, minimum received power threshold, $P_{M I N j}$ in constraint (5e) is increased by $\delta=1 \mathrm{~dB}$ and the MIQP model is solved for this new setting. The number of active UEs that are randomly placed in the considered playground has been set variable from 5 to 230 UEs. The maximum value has been chosen according to the maximum number of UEs that can be managed by the Closest $B S$ mapping solution without any capacity outage, which gives the maximum load of the cellular network.

The results are obtained by statistical analysis considering 50 simulation runs and a 95\% confidence interval. Fig. 1(a) presents the comparison of the number of active BSs for MinPower-QoS with respect to the other solutions, i.e., Closest BS mapping and MinPower. From the figure the behaviour of proposed solution is evident: if it is feasible, for each number of active UEs the minimum power configuration is selected; note that the number of active BSs is almost linearly dependent on the number of UEs. Because of the QoS requirements, the slope of MinPower-QoS is higher than MinPower. Finally, the MinPower-QoS converges to Closest $B S$ mapping when the number of active users increases. This result is reinforced by Fig. 1(b) where total power consumption is depicted versus the number of users. It is interesting to see that in the interference limited scenario under high load, all BSs are activated but MinPower-QoS can still have power savings over the Closest BS mapping due to its flexibility in user association and bandwidth distribution. Moreover, the performance of MinPower-QoS is close to the global optimum which is represented by MinPower when the number of users is very low.

More in detail, the bandwidth impact in MinPower-QoS optimization can be highlighted. The solutions assigning the bandwidth prioritizing UEs with lower signal quality, i.e. MinPower-QoS no res and MinPower-QoS res, perform better from the power consumption perspective. Such a behaviour can be explained by a more flexible management of the radio resources that allows a lower number of active BSs and a lower transmission power. In Fig. 1(c) the UE satisfaction rate is depicted starting from optimum MinPower solution as first iteration. From this figure, it is possible to note the impact of the number of active UEs on the number of required iterations to reach 0 outages. Finally, it is possible to see that, while MinPower experiences outages, MinPower-QoS converges to 0 outage performance after a certain number of iterations depending on the number of UEs. Each iteration corresponds to a solution in the search space for the MIQP model which stops when it reaches a minimum power solution with 0 outages. The average transmission power vs the number of iterations is depicted in Fig. 1(c). Active UEs are satisfied when mapping and received power allow to reach the target QoS. Among the feasible solutions, the one minimizing the 


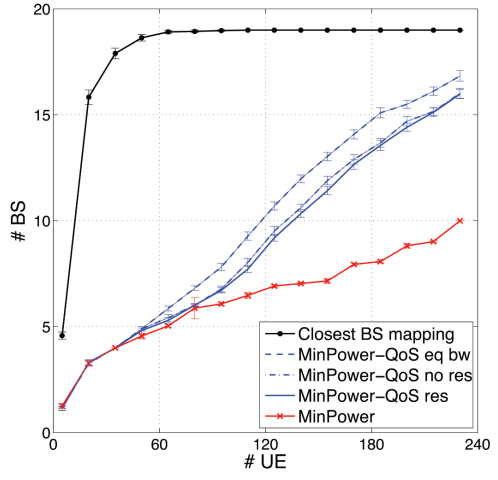

(a)

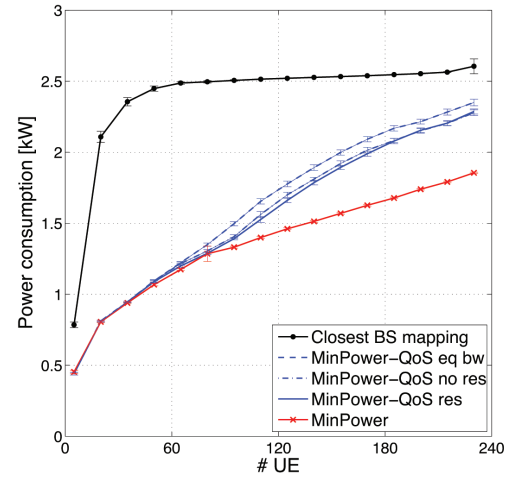

(b)

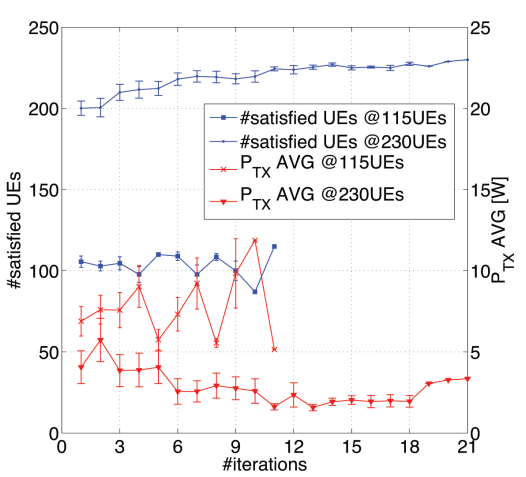

(c)

Fig. 1. Simulation results: (a) active BSs vs number of UEs; (b) global power consumption vs number of UEs; (c) satisfied UEs and average transmission power per active BS vs number of iterations in MinPower-QoS

global power consumption is chosen. Particularly, even if the proper mapping is obtained by increasing the BS transmission powers, this trend can also be harmful for some UEs at the cell edge because of the interference level. Therefore, the BSs can also be switched on, reducing the total power consumption together with the interference caused by very high transmission power.

The fluctuations in Fig. 1(c) are not related to any optimization variable and the algorithm stability is not affected by this result. On the other hand a lower number of UEs requires less iterations to reach the solution.

\section{CONCLUSION}

In this paper, a novel optimization framework is developed which is aimed at minimizing the power consumption with guaranteed QoS. The results for interference limited scenarios and different traffic loads are shown. By putting the cells in sleep mode, up to $60 \%$ power savings can be achieved with respect to the basic scheme. Moreover, the proposed MinPower-QoS methodology affords performance very close to the optimum solution, particularly for low traffic load scenarios. This study demonstrates the potential savings by long term sleep in a multi cell scenario. Significant savings happen when the traffic is below $35 \%$ of the maximum load. Above that level, in medium to high traffic load, other energy efficiency features can be preferred instead of deep sleep. Combination of different energy saving features is considered as a future work.

\section{REFERENCES}

[1] "Cisco visual networking index: Global mobile data traffic forecast update, http://www.cisco.com/."

[2] F. Richter, A. Fehske, and G. Fettweis, "Energy efficiency aspects of base station deployment strategies for cellular networks," in Vehicular Technology Conference Fall (VTC 2009-Fall), 2009 IEEE 70th, 2009, pp. $1-5$.

[3] M. Marsan, L. Chiaraviglio, D. Ciullo, and M. Meo, "Switch-off transients in cellular access networks with sleep modes," in Communications Workshops (ICC), 2011 IEEE International Conference on, June 2011, pp. 1-6.
[4] P. Frenger, P. Moberg, J. Malmodin, Y. Jading, and I. Godor, "Reducing energy consumption in LTE with cell DTX," in Vehicular Technology Conference (VTC Spring), 2011 IEEE 73rd, May 2011, pp. 1-5.

[5] T. Chen, Y. Yang, H. Zhang, H. Kim, and K. Horneman, "Network energy saving technologies for green wireless access networks," Wireless Communications, IEEE, vol. 18, no. 5, pp. 30-38, October 2011.

[6] S. Videv and H. Haas, "Energy-efficient scheduling and bandwidthenergy efficiency trade-off with low load," in Communications (ICC), 2011 IEEE International Conference on, June 2011, pp. 1-5.

[7] L. Venturino, C. Risi, A. Zappone, and S. Buzzi, "Green joint user scheduling and power control in downlink multi-cell OFDMA networks," in Future Network and Mobile Summit (FutureNetworkSummit), 2013, July 2013, pp. 1-10.

[8] C. Y. Wong, R. Cheng, K. Lataief, and R. Murch, "Multiuser OFDM with adaptive subcarrier, bit, and power allocation," Selected Areas in Communications, IEEE Journal on, vol. 17, no. 10, pp. 1747-1758, Oct 1999.

[9] A. Yildiz, T. Girici, and H. Yanikomeroglu, "A pricing based algorithm for cell switching off in green cellular networks," in Vehicular Technology Conference (VTC Spring), 2013 IEEE 77th, June 2013, pp. 1-6.

[10] H. Holtkamp, G. Auer, S. Bazzi, and H. Haas, "Minimizing base station power consumption," Selected Areas in Communications, IEEE Journal on, vol. 32, no. 2, pp. 297-306, February 2014.

[11] X. Xiao, X. Tao, and J. Lu, "QoS-Guaranteed Energy-Efficient Power Allocation in downlink multi-user MIMO-OFDM systems," in Cотmunications (ICC), 2014 IEEE International Conference on, June 2014, pp. 3945-3950.

[12] Z. Ren, S. Chen, B. Hu, and W. Ma, "Energy-Efficient Resource Allocation in Downlink OFDM Wireless Systems With Proportional Rate Constraints," Vehicular Technology, IEEE Transactions on, vol. 63, no. 5, pp. 2139-2150, Jun 2014.

[13] M. Olsson, C. Cavdar, P. Frenger, S. Tombaz, D. Sabella, and R. Jantti, "5GrEEn: Towards Green 5G mobile networks," in Wireless and Mobile Computing, Networking and Communications (WiMob), 2013 IEEE 9th International Conference on, Oct 2013, pp. 212-216.

[14] G. Auer, V. Giannini, C. Desset, I. Godor, P. Skillermark, M. Olsson, M. Imran, D. Sabella, M. Gonzalez, O. Blume, and A. Fehske, "How much energy is needed to run a wireless network?" Wireless Communications, IEEE, vol. 18, no. 5, pp. 40-49, October 2011.

[15] J. Zander, S.-L. Kim, M. Almgren, and O. Queseth, Radio Resource Management for Wireless Networks. Norwood, MA, USA: Artech House, Inc., 2001.

[16] "Further advancements for E-UTRA physical layer aspects v.9.0.0," 3GPP TR 36.814, 2010.

[17] K. Hiltunen, "Utilizing enodeb sleep mode to improve the energyefficiency of dense lte networks," in Personal Indoor and Mobile Radio Communications (PIMRC), 2013 IEEE 24th International Symposium on, Sept 2013, pp. 3249-3253. 